\title{
Efficient Brain Tumor Detection Using Wavelet Transform
}

\author{
Ku. Mayuri R. Khode*, Prof. S. R. Salwe**, Prof. A.P. Bagade***, \\ Dr. R. D. Raut**** \\ *(M.Tech.VLSI student, B.D.C.E, Sewagram.) \\ **(EXTC Department, B.D.C.E, Sewagram.) \\ ***(EXTC Department, B.D.C.E, Sewagram.) \\ ****(Electronics Deapartment, B.D.C.E., Sewagram.)
}

\begin{abstract}
Brain tumor detection is a challenging task and its very important to analyze the structure of the tumor correctly so a automatic method is used now a days for the detection of the tumor. This method saves time as well as it reduces the error which occurs in the method of manual detection. In this paper the tumor is detected using wavelet transform. MRI is an important tool used in many fields of medicine and is capable of generating a detailed image of any part of the human body. The tumor is segmented from the MRI images, features are extracted and then the area of the tumor is determined. PNN can successfully handle the process of brain tumor classification.
\end{abstract}

Keywords: Brain tu mor, detection, MRI, segment, wavelet.

\section{INTRODUCTION}

The buildup of extra cells often forms a mass of tissue called a growth or tumor. Brain tumor is one of the major causes of death among people. The symptoms of a brain tumor depend on tumor size, type and location. Symptoms may be caused when a tumor presses on a nerve or harms a part of a brain. Also they may be caused when a tumor blocks the fluid that flows through and around the or when the brain swells because build up of fluid. Headaches, nausea and vomiting, Changes in speech, vision or hearing, problem balancing or walking, changes in mood, personality or ability to concentrate, problems with memory, muscle jerking or itching, nu mbness or tingling in the arms or legs.

Accurate detection of the type of brain abnormality is highly essential for treatment planning which can minimize the fatal results. Manual detection of brain tumor is a tedious job and takes a lot of time and not accurate, varies from one doctor to another. Accurate results can be obtained only through computer aided automated systems. Besides being accurate, these techniques must coverage quickly in order to apply them for real time applications. Brain tumor can be diagnosed by using magnetic resonance imaging (MRI), ultrasonic, CT images and X-rays. Magnetic Resonance Imag ing is an important tool used in many fields of medicine and is capable of generating a detailed image of any part of the human body.

MRI stands for Magnetic Resonance Imaging. A MRI scanner uses powerful magnets to polarise and excite hydrogen nuclei (single proton) in human tissue, which produces a signal that can be detected and it is encoded spatially, resulting in images of the body. The MRI machine emits radio frequency (RF) pulse that specifically binds only to hydrogen. The system sends the pulse to that specific area of the body that needs to be examined. Due to the RF pulse, protons in that area absorb the energy needed to make them spin in a different direction. This is meant by the resonance of MRI. The RF pulse makes the protons spin at the larmour frequency, in a specific direction. This frequency is found based on the particular tissue being imaged and the strength of the main magnetic field.

Classification of the brain tumor is also a important task for treatment planning. There are two types of tumor which are- benign (non-cancerous) and malignant (cancerous) tumors. Conventional methods involve invasive techniques such as biopsy, lu mbar puncture and signal tap method, to detect and classify brain tumor into benign and malignant which are very painful and time consuming.

Wavelet analysis is an effective methodology capable of revealing aspects of data which other signal analysis techniques. Analyzing the images at multiple levels, the method is able to extract finer details from them and in turn improves the quality of the image. In addition, wavelet analysis is capable of compressing or de-noising a signal without appreciable degradation. Wavelet analysis is of at most importance in case of delicate information, such as in case of medical imaging. 


\section{IMPLIMENTED WORK}

2.1 MR image to gray scale image:

MRI images are magnetic resonance images which can be acquired on computer when a patient is scanned by MRI machine. We can acquire MRI images of the part of the body which is under test or desired. Generally when we see MRI images on computer they looks like black and white images. In analog practice, gray scale imaging is sometimes called "black and white," but technically this is a misnomer. In true black and white, also known as halftone, the only possible shades are pure black and pure white. The illusion of gray shading in a halftone image is obtained by rendering the image as a grid of black dots on a white background (or vice versa), with the sizes of the individual dots determining the apparent lightness of the gray in their vicinity. The lightness of the gray is directly proportional to the number representing the brightness levels of the primary colors. Black is represented by $R=G=B=$ 0 or $R=G=B=00000000$, and white is represented by $\mathrm{R}=\mathrm{G}=\mathrm{B}=255$ or $\mathrm{R}=\mathrm{G}=\mathrm{B}=11111111$.

Grayscale is a range of shades of gray without apparent color. The darkest possible shade is black, which is the total absence of transmitted or reflected light. The lightest possible shade is white, the total transmission or reflection of light at all visible wavelengths. So because of the above reasons first we convert our MRI image to be preprocessed in grayscale image.

\subsection{Image segmentation:}

In image segmentation the level to which the subdivision of an image into its constituent regions or objects is carried depends on the problem being solved. In other words, when the object of focus is separated, image segmentation should stop the main goal of segmentation is to divide an image into parts having strong correlation with areas of interest in the image. In medical imaging for analyzing anatomical structures such as bones, muscles blood vessels, tissue types, pathological regions such as cancer, multiple sclerosis lesions and for dividing an entire image into sub regions such as the white matter (WM), gray matter (GM) and cerebrospinal fluid (CSF) spaces of the brain automated delineation of different image components are used. In the field of medical image processing segmentation of MR brain image is significant as MRI is particularly suitable for brain studies because of its excellent contrast of soft is sues, non invasive characteristic and a high spatial resolution Segmentation is the process of splitting an observed image into its homogeneous or constituent regions. The goal of segmentation is to simplify or change the representation of an image into something that is more meaningful and easier to analyze. It is important in many computer vision and image processing application.

Fuzzy C-means clustering is the overlapping clustering technique. One pixel value depending upon the two or more clusters centers. It is also known as soft clustering method. Most widely used the fuzzy clustering algorithms is the Fuzzy Cmeans (FCM) algorith $\mathrm{m}$. The FCM algorith $\mathrm{m}$ is the partition of the $n$ element $X=\{x 1, \ldots, x n\}$ into a collection of the $\mathrm{c}$ fuzzy clustering with respect to the below given criteria. It is based on the minimization of the following objective function:

$\mathrm{J}=\sum_{i=1}^{N} \sum_{j=1}^{c} u_{i j}^{m}\left|x_{i}-y_{i}\right|^{2}$

Where, $\mathrm{m}=$ level of the fuzziness and real number greater than 1 .

$\mathrm{uij}=$ degree of the membership of $\mathrm{xi}$ in the cluster $\mathrm{cj}$ $\mathrm{x}=$ data value

Fuzzy C-means is the popular method for medical image segmentation but only consider the image intensity thereby producing unsatisfactory results in noisy images. A bunch of the algorithms are proposed to make the FCM robust against noise and in homogeneity but it's still not perfect. fuzzy cmean is slower than the K-means in efficiency but gives the accurate prediction of tumor cells which are not predicted by the K-means algorith $\mathrm{m}$.

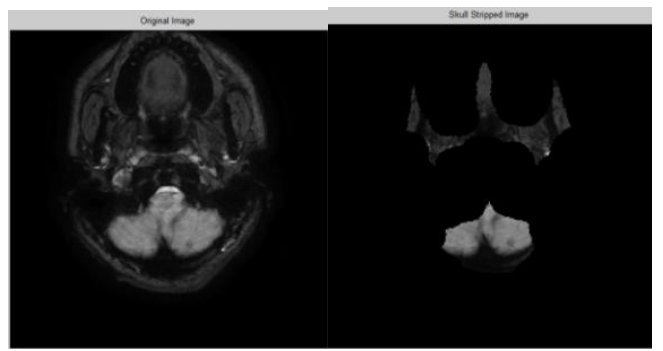

Original image Skull stripped image

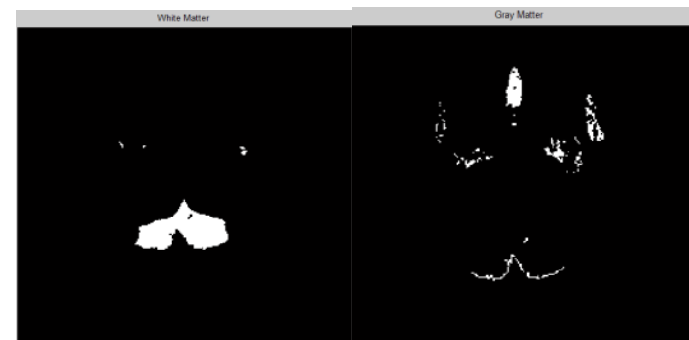

White matter

Gray matter

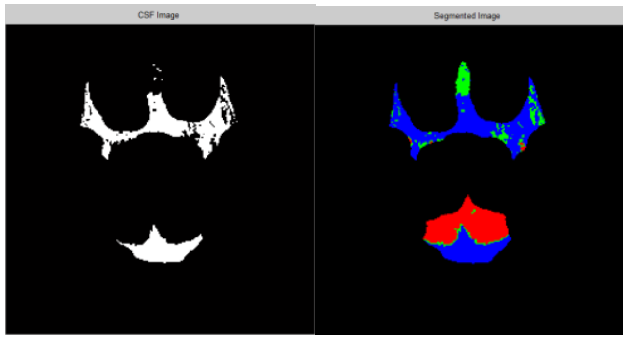

CSF matter Segmented image 


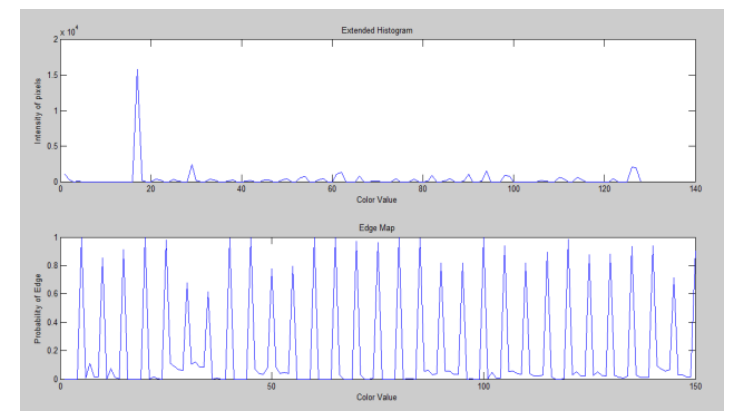

Fig: Extended Histogram and Edge map

\subsection{Create database:}

A database is an organized collection of data and here it's a collection of images. The database is created using a number of images that are selected and divided into two groups that are healthy and disease image.
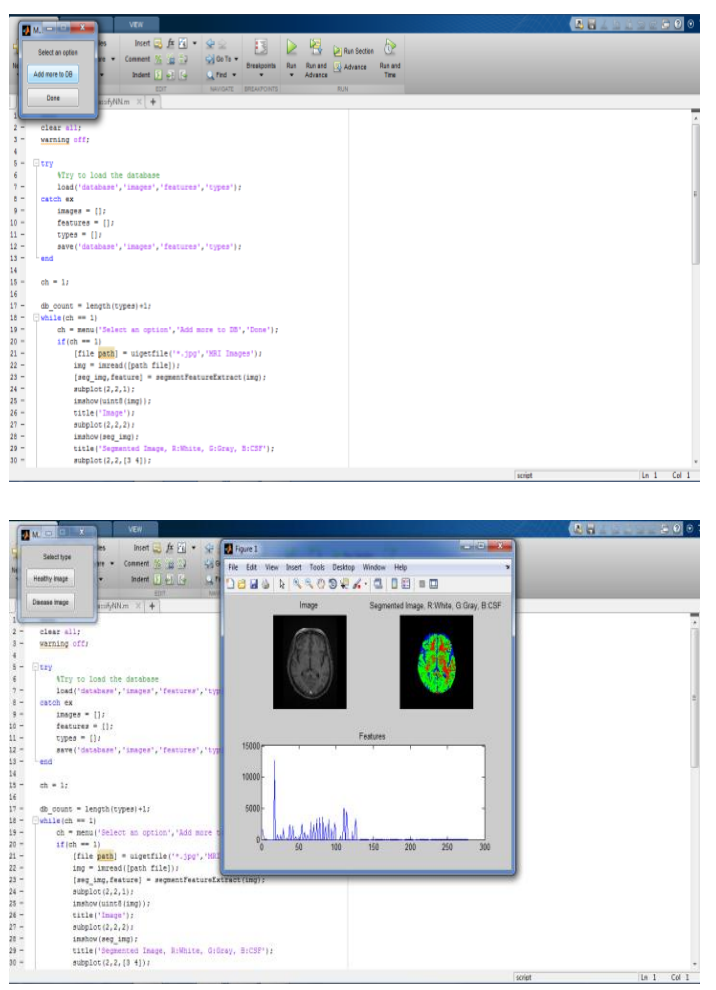

\subsection{Feature extraction:}

Texture analysis makes differentiation of normal and abnormal tissue easy. It even provides contrast between malignant and normal tissue, which may be below the threshold of human perception. Texture analysis using computer aided diagnosis can be used to replace biopsy techniques and plays an important role in early diagnosis and tracking of diseases.

The wavelet is a powerful mathematical tool for feature extraction, and has been used to extract the wavelet coefficients from MR images. Discrete Wavelet Transform is an implementation of the WT using the dyadic scales and positions.
Two dimensional DWT results in four sub bands LL (low-low), LH (low-high), HL (high-low), $\mathrm{HH}$ (high-high) at each scale. Sub band LL, is the approximation component of the image, which is used for next two dimensional DWT. Whereas, LH, $\mathrm{HL}, \mathrm{HH}$ are the detailed components of the image along the horizontal, vertical and diagonal axis, respectively, as shown in the figure:

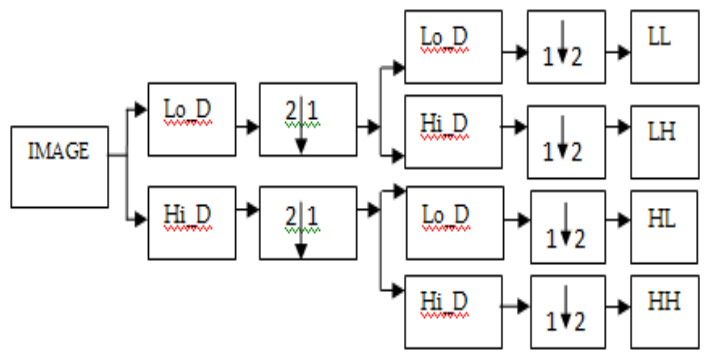

Lo_D: low pass filter

Hi_D: high pass filter

$$
2 \downarrow 1-\text { Down sampling columns: keeps }
$$

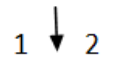

- Down sampling rows: keeps the even indexed rows.

\subsection{Classification:}

Classification is done using probabilistic neural network. Probabilistic Neural Network (PNN) is a Radial Basis Neural Network, which provides a general solution to pattern classification problems by following an approach developed in statistics, called Bayesian clas sifiers.

Probabilistic Neural Network has three layers, as shown in fig., the Input layer, Radial Basis Layer and the Competitive Layer. Radial Basis Layer evaluates vector distances between input vector and row weight vectors in weight matrix. These distances are scaled by Radial Basis Function nonlinearly. Then the Competitive Layer finds the shortest distance among them, and thus finds the training pattern closest to the input pattern based on their distance.

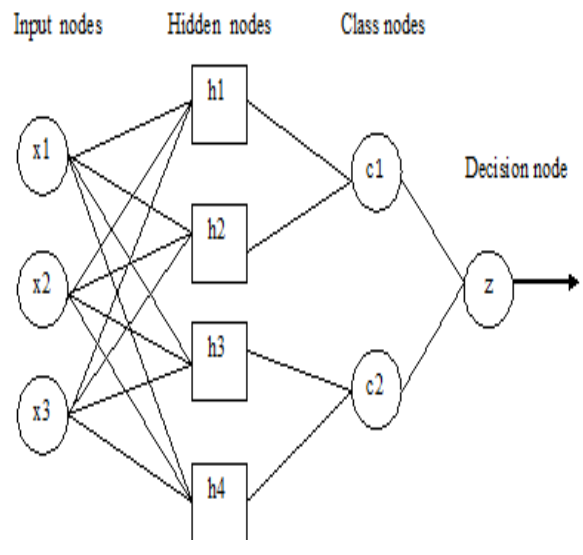



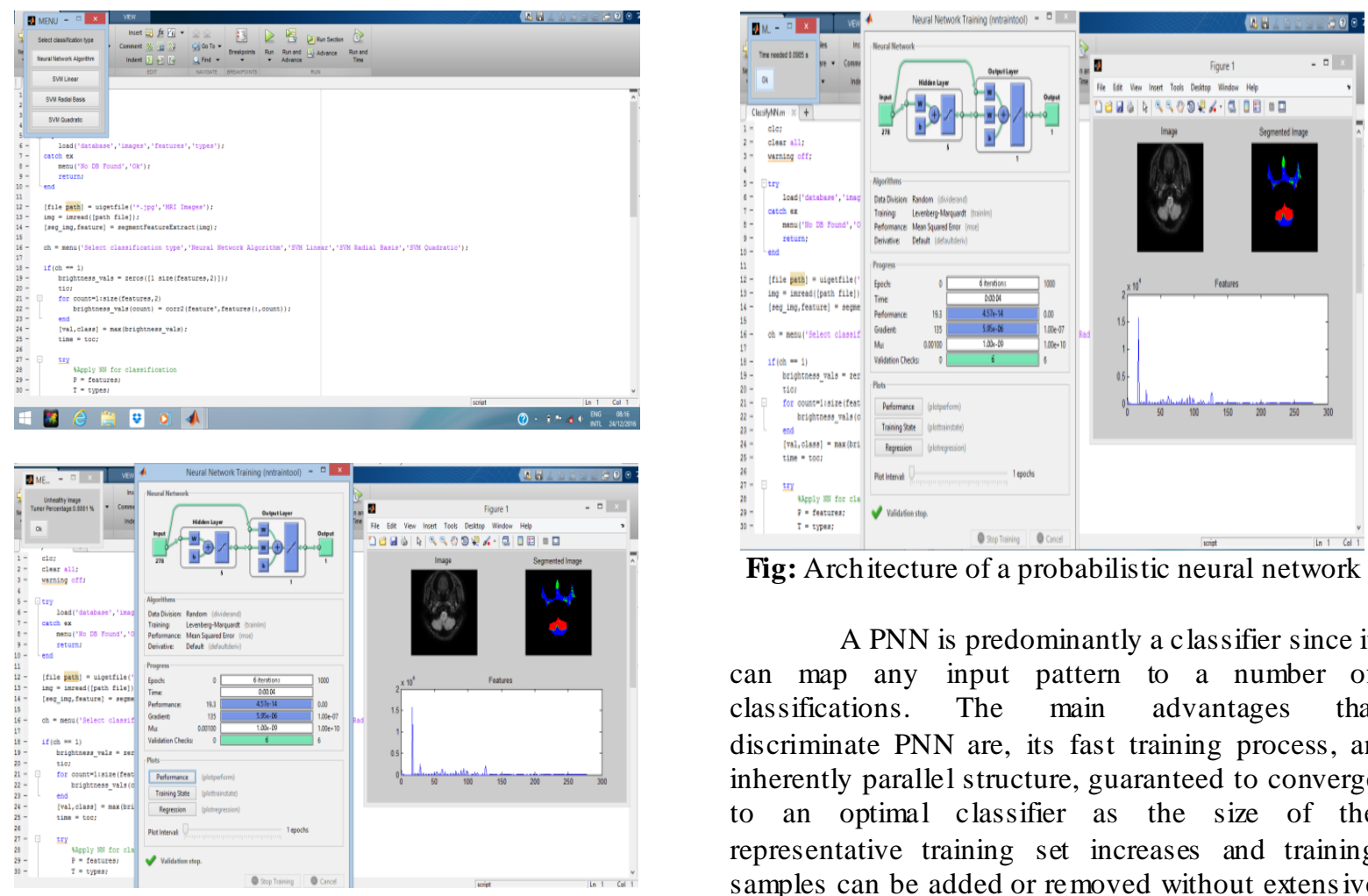

Fig: Arch itecture of a probabilistic neural network

A PNN is predominantly a classifier since it can map any input pattern to a number of classifications. The main advantages that discriminate PNN are, its fast training process, an inherently parallel structure, guaranteed to converge to an optimal classifier as the size of the representative training set increases and training samples can be added or removed without extensive retrain ing.

Table: Comparison of four different methods based on time required for the image processing:

\begin{tabular}{|l|l|l|l|l|l|}
\hline Images & $\begin{array}{l}\text { Neural } \\
\text { network } \\
\text { Algorithm }\end{array}$ & $\begin{array}{l}\text { SVM Linear } \\
\text { (Time in sec) }\end{array}$ & $\begin{array}{l}\text { SVM Radial } \\
\text { Basis } \\
\text { in sec) }\end{array}$ & $\begin{array}{l}\text { SVM } \\
\text { Quadratic } \\
\text { (Time in sec) }\end{array}$ & Tumor \% \\
\hline 1. & $0.0573 \mathrm{~s}$ & $1.4350 \mathrm{~s}$ & $1.6368 \mathrm{~s}$ & $0.4680 \mathrm{~s}$ & $0.8001 \%$ \\
\hline 2. & $0.0035 \mathrm{~s}$ & $0.1483 \mathrm{~s}$ & $0.5657 \mathrm{~s}$ & $0.5095 \mathrm{~s}$ & $2.032 \%$ \\
\hline 3. & $0.0050 \mathrm{~s}$ & $0.1528 \mathrm{~s}$ & $0.5787 \mathrm{~s}$ & $0.5339 \mathrm{~s}$ & $1.1444 \%$ \\
\hline 4. & $0.1178 \mathrm{~s}$ & $0.1528 \mathrm{~s}$ & $1.5465 \mathrm{~s}$ & $0.5393 \mathrm{~s}$ & $1.6780 \%$ \\
\hline 5. & $0.0867 \mathrm{~s}$ & $1.1615 \mathrm{~s}$ & $1.0087 \mathrm{~s}$ & $0.4699 \mathrm{~s}$ & $0.8713 \%$ \\
\hline 6. & $0.0374 \mathrm{~s}$ & $0.1424 \mathrm{~s}$ & $0.4937 \mathrm{~s}$ & $0.4622 \mathrm{~s}$ & $1.5935 \%$ \\
\hline 7. & $0.0039 \mathrm{~s}$ & $0.1545 \mathrm{~s}$ & $0.5315 \mathrm{~s}$ & $0.4689 \mathrm{~s}$ & $1.3418 \%$ \\
\hline 8. & $0.0040 \mathrm{~s}$ & $0.1439 \mathrm{~s}$ & $0.5119 \mathrm{~s}$ & $0.5073 \mathrm{~s}$ & $1.8031 \%$ \\
\hline 9. & $0.0036 \mathrm{~s}$ & $0.1456 \mathrm{~s}$ & $0.4946 \mathrm{~s}$ & $0.4599 \mathrm{~s}$ & $1.5274 \%$ \\
\hline 10. & $0.0040 \mathrm{~s}$ & $0.1444 \mathrm{~s}$ & $0.4965 \mathrm{~s}$ & $0.4665 \mathrm{~s}$ & $2.0335 \%$ \\
\hline 11. & $0.0035 \mathrm{~s}$ & $0.1438 \mathrm{~s}$ & $0.5232 \mathrm{~s}$ & $0.4738 \mathrm{~s}$ & $1.5340 \%$ \\
\hline 12. & $0.0035 \mathrm{~s}$ & $0.1487 \mathrm{~s}$ & $0.5099 \mathrm{~s}$ & $0.4625 \mathrm{~s}$ & $2.4760 \%$ \\
\hline 13. & $0.0035 \mathrm{~s}$ & $0.1476 \mathrm{~s}$ & $0.6110 \mathrm{~s}$ & $0.5326 \mathrm{~s}$ & $1.5869 \%$ \\
\hline 14. & $0.0047 \mathrm{~s}$ & $0.1471 \mathrm{~s}$ & $0.5136 \mathrm{~s}$ & $0.4611 \mathrm{~s}$ & $2.2949 \%$ \\
\hline 15. & $0.0037 \mathrm{~s}$ & $0.1562 \mathrm{~s}$ & $0.5153 \mathrm{~s}$ & $0.4796 \mathrm{~s}$ & $1.5406 \%$ \\
\hline 16. & $0.0036 \mathrm{~s}$ & $0.1416 \mathrm{~s}$ & $0.5275 \mathrm{~s}$ & $0.4639 \mathrm{~s}$ & $2.1851 \%$ \\
\hline 17. & $0.0035 \mathrm{~s}$ & $0.1445 \mathrm{~s}$ & $0.5248 \mathrm{~s}$ & $0.4561 \mathrm{~s}$ & $0.2218 \%$ \\
\hline 18. & $0.0044 \mathrm{~s}$ & $0.1423 \mathrm{~s}$ & $0.4995 \mathrm{~s}$ & $0.4730 \mathrm{~s}$ & $1.7431 \%$ \\
\hline 19. & $0.0038 \mathrm{~s}$ & $0.1672 \mathrm{~s}$ & $0.4922 \mathrm{~s}$ & $0.4752 \mathrm{~s}$ & $1.5630 \%$ \\
\hline 20. & $0.0037 \mathrm{~s}$ & $0.1438 \mathrm{~s}$ & $0.5139 \mathrm{~s}$ & $0.4666 \mathrm{~s}$ & $1.6469 \%$ \\
\hline 21. & $0.0036 \mathrm{~s}$ & $0.1448 \mathrm{~s}$ & $0.5164 \mathrm{~s}$ & $0.4746 \mathrm{~s}$ & $0.6470 \%$ \\
\hline 22. & $0.0035 \mathrm{~s}$ & $0.1425 \mathrm{~s}$ & $0.4978 \mathrm{~s}$ & $0.4629 \mathrm{~s}$ & $1.6713 \%$ \\
\hline 23. & $0.0048 \mathrm{~s}$ & $0.1442 \mathrm{~s}$ & $0.5077 \mathrm{~s}$ & $0.4729 \mathrm{~s}$ & $1.3667 \%$ \\
\hline 24. & $0.0035 \mathrm{~s}$ & $0.1587 \mathrm{~s}$ & $0.5056 \mathrm{~s}$ & $0.4892 \mathrm{~s}$ & $1.6795 \%$ \\
\hline 25. & $0.0036 \mathrm{~s}$ & $0.1444 \mathrm{~s}$ & $0.5189 \mathrm{~s}$ & $0.4584 \mathrm{~s}$ & $0.1221 \%$ \\
\hline & & & & & \\
\hline
\end{tabular}




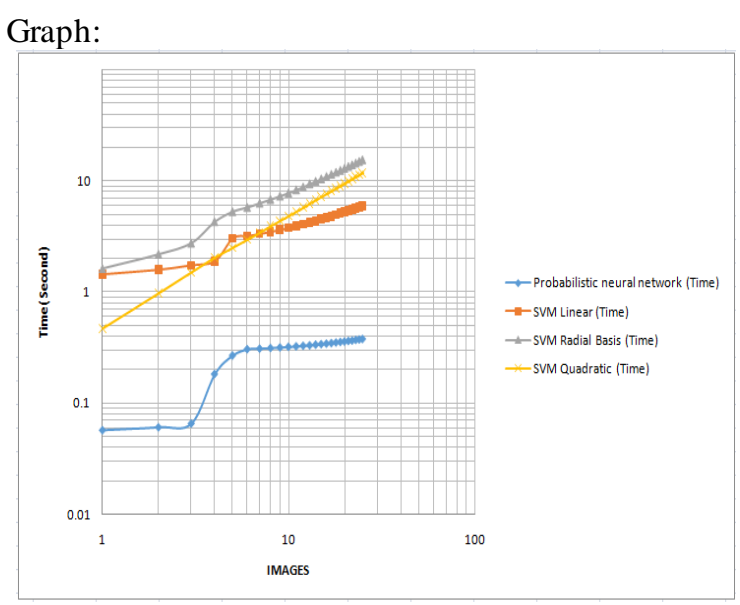

Fig: Time taken by four methods

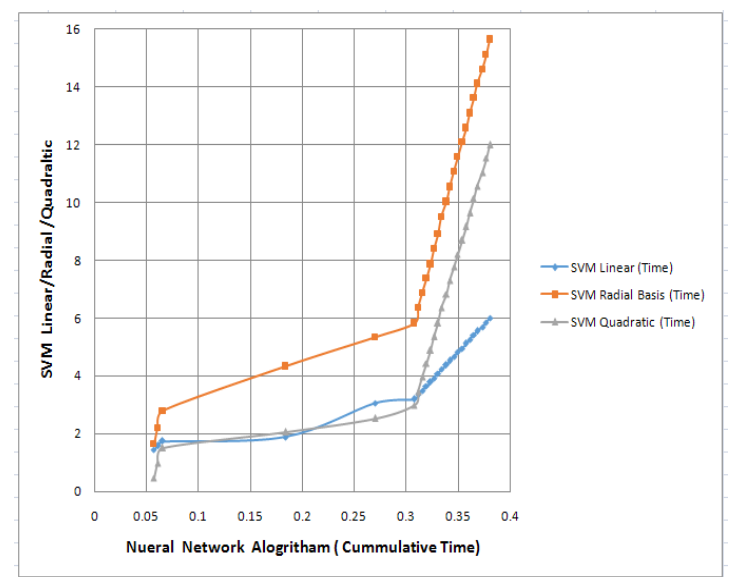

Fig: Cummu lative time comparis on of the four methods

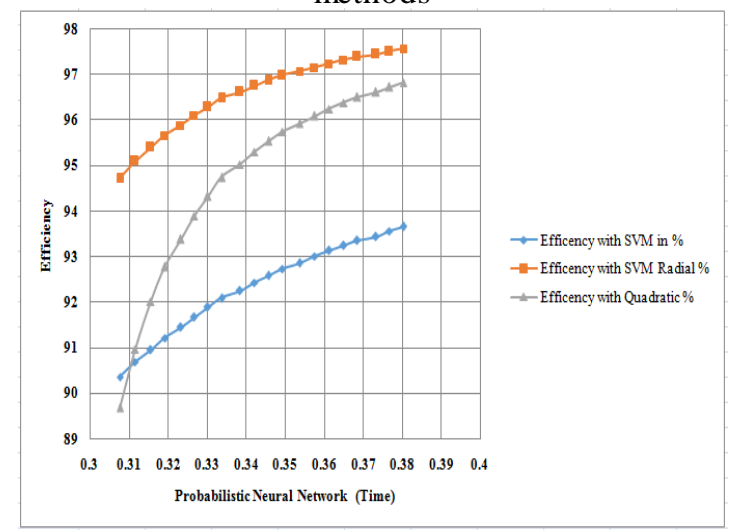

Fig: Comparison of efficiency of PNN with the other three methods

\section{FUTURE SCOPE}

The goal is to detect, to segment, and to identify most types of pathological tissue that occur within pediatric brain tumors. Future research in the segmentation of medical images will lead towards improving the accuracy, exactness, and computational speed of segmentation approaches, as well as minimizing the amount of time taken for the process.

\section{CONCLUSION}

In this project fuzzy c-mean clustering algorithm is used for segmentation methods, classification of brain tumor and its feature extraction is done using probabilistic neural network algorith $\mathrm{m}$. Many algorith ms have been proposed in the literature for each processing stage. So this method used gives the better result. The results are given and the graph showing the comparison in terms of time given by various methods that is neural network, SVM linear, SVM radial basis, SVM quadratic is given.

\section{REFERENCES}

[1]. Ahmed Kharrat, Nacéra Benamrane, Abid Mariem "Detection of Brain Tumor Medical Images" International Conference on Signals, Circuits and Systems 2009.

[2]. Dr. M. Karnan, K. Selvanayaki "Improved Implementation of Brain MR Image Segmentation Using Meta Heuristic Algorithms" 2: Research Scholar 978-14244-5967-4/10/\$26.00 @2010 IEEE.

[3]. P. Padilla, J. M. Gorriz, J. Ramirez, R. Chaves "Alzheimer's disease detection in functional images using 2D Gabor Wavelet analysis" Electronics letter, vol. 46 no. $815^{\text {th }}$ April 2010.

[4]. Sudipta Roy, Atanu Saha, Samir K. Bandyopadhyay "Brain tumor segmentation and quantification from MRI of brain" Journal of global research in computer science, volume 2, No. 4, April 2011.

[5]. Sudipta Roy, Samir K. Bandyopadhyay "Detection and Quantification of Brain Tumor from MRI of brain and its Symmetric Analysis" International Journal of Information and Communication Technology Research Volume 2 No. 6, June 2012.

[6]. Arati Kothari "Detection and classification of brain cancer using artificial neural network in MRI image" World journal of science and technology 2012.

[7]. S. Karpagam and S. Gowri "Brain Tumor Growth and Volume Detection by Ellipsoid-Diameter Technique Using MRI Data" IJCSI International Journal of Computer Science Issues, Vol. 9, Issue 2, No 1, March 2012.

[8]. Pauline John "Brain Tumor Classification Using Wavelet and Texture Based Neural Network" International Journal of Scientific \& Engineering Research Volume 3, Issue 10, October-2012.

[9]. Kailash Sinha, G. R. Sinha "Efficient Segmentation Methods for Tumor 
Detection in MRI Images " IJCSI International Journal of Computer Science Issues, Vol. 9, Issue 2, No 1, March 2012.

[10]. Smita Haribhau Zol "Analytical study of segmentation and quantification of brain tumor and its importance in radiology" Vol. 1No.Jan 2012.

[11]. Snehalatha and Dr. Narendra Mustare "Computer Based Detection and Classification Technique for Brain Cancer" International Journal of Research in Engineering and Technology (IJRET) Vol. 1, No. 5, 2012.

[12]. Prof. B. K. Saptalakar, Miss. Rajeshwari. H "Segmentation Based Detection of Brain Tumor" International Journal of Computer and Electronics Research [Volume 2, Issue 1, February 2013].

[13]. Vivek Angoth, CYN Dwith, A marjot Singh "A Novel Wavelet Based Image Fusion for Brain Tumor Detection" International Journal of Computer Vision and Signal Processing, 2(1), 1-7(2013).

[14]. Kimmi Verma, Aru Mehrotra, Vijayeta Pandey, Shardendu Singh "Image Processing Techniques For The Enhancement Of Brain Tumor Patterns" International Journal of Advanced Research in Electrical, Electronics and Instrumentation Engineering Vol. 2, Issue 4, April 2013.

[15]. Jay Patel and Kaushal Doshi "A Study of Segmentation Methods for Detection of Tumor in Brain MRI" Advance in Electronic and Electric Engineering ,Volume 4, Number 3 (2014).

[16]. Tarun Kumar Agarwal, Mayank Tiwari, Subir Singh Lamba "Modified Histogram Based Contrast Enhancement using Homomorphic Filtering for Medical Images" IEEE International Advance Computing Conference (IACC) 2014.

[17]. Rohini Paul Joseph, C. Senthil Singh, M.Manikandan "Brain Tumor MRI Image Segmentation And Detection In Image Processing" IJRET International Journal of Research in Engineering and Technology Mar-2014.

[18]. Ed-Edily Mohd. Azhari, Muhd. Mudzakkir Mohd. Hatta, Zaw Zaw Htike and Shoon Lei Win "Brain Tumor Detection And Localization In Magnetic Resonance Imaging" International Journal of Information Technology Convergence and Services (IJITCS) Vol.4, No.1, February 2014.

[19]. CH. Rambabu, B. Siva Ayyappa "Brain Tumor Classification Using Multi Wavelet
Transform and Neural Network” International Journal of Advanced Research in Computer Science and Software Engineering Volume 4, Issue 9, September 2014.

[20]. Anisha M. Lal, M. Balaji, D. Aju "MultiLevel Fusion of CT and MRI Brain Images for Classifying Tumor"International Journal of Enhanced Research in Management \& Computer Applications Vol. 3 Issue 8, August 2014. 